\title{
Estimative of Wooden Toughness by the Apparent Density and Bending Strength
}

\author{
André Luis Christoforo ${ }^{1, *}$, Sérgio Augusto Mello da Silva ${ }^{2}$, Tulio Hallak Panzera ${ }^{3}$, \\ Francisco Antonio Rocco Lahr ${ }^{4}$
}

\author{
${ }^{1}$ Department of Civil Engineering, Federal University of São Carlos, São Carlos - SP, 13565-905, Brazil \\ ${ }^{2}$ Department of Civil Engineering, Paulista State University (UNESP), Ilha Solteira - SP, 15.385-000, Brazil \\ ${ }^{3}$ Department of Mechanical Engineering, Federal University of São João del-Rei, São João del-Rei - MG, 36307-352, Brazil \\ ${ }^{4}$ Department of Structural Engineering, University of São Paulo (EESC/USP), São Carlos - SP, 13565-905, Brazil
}

\begin{abstract}
The wooden toughness is a mechanical property of interest in the project in the case of the design of structures and structural elements subjected to impact loads, however, not an integral part of the mechanical properties commonly investigated, such as the modulus of elasticity and strength modulus in tensile, in bending and others. In order to corroborate with the most knowledge about the toughness of wood, this study aimed to investigate the possibility of estimating the toughness by the apparent density and strength in bending, using linear, quadratic and cubic regression models. Were used 15 species of wood, equally distributed in the five strength classes defined by the Brazilian standard ABNT NBR 7190:1997, enabling greater coverage of the results to estimate the toughness of wood for various species. The results of the regression models showed to be representative all the functions evaluated, presenting the quadratic fits in function of the apparent density the best results.
\end{abstract}

Keywords Toughness, Wood, Regression models, Apparent density, Bending strength

\section{Introduction}

The stress caused by the impact loads acts only in a very short time interval, for example, a few microseconds [1]. A structural element of wood is considered tough one that supports large deformation under bending or twisting before fracture [2].

Usually has restricted the use of the toughness term to a particular mechanical property, to qualify the wood under the action of an impact load causing failure. A material that offers improved strength to failure than others will have a greater toughness value, according to Van Vlack [3]. For wood, shows high values of toughness in comparison with other materials. This makes it particularly useful for applications where the material is subjected to impact loads, according to Schneider et al. [4].

Toughness has been used to describe characteristics of the wood and its derivatives, being related to other properties such as shear strength and tensile strength in perpendicular to the grain. However, the toughness should not be considered separately to characterize a species, requiring the evaluation of the results of other tests [3].

* Corresponding author:

alchristoforo@yahoo.com.br (André Luis Christoforo)

Published online at http://journal.sapub.org/ijme

Copyright (C) 2014 Scientific \& Academic Publishing. All Rights Reserved
In addition, changes in the values of toughness are good indicators of damage or abnormalities in structural lumber. The relationship between toughness and anatomical characteristics of the wood have been the subject of detailed examination, as records Van Vlack [3].

In general, the greater knowledge of the physical and mechanical properties of wood allows their best achievement [5-7]. Because of difficulties in performing tests for the characterization of the species, the wood is often used without the basic knowledge of their properties, thus leading to wastage of materials [8].

Special importance is on the study of wood in relation to its performance when applied to loads that cause impact [9], motivating the development of research on this topic.

Pazos et al. [10] investigated the toughness and impact strength of Lysiloma bahamensis wood, density $0.62 \mathrm{~g} / \mathrm{cm}^{3}$, under dry air $(12 \%)$, saturated. The values of toughness or work absorbed (W) obtained from saturated and dry woods were equal to $41.75 \mathrm{~J}$ and $46.75 \mathrm{~J}$, respectively. Among others, the authors concluded that the saturated wood showed the highest toughness and impact strength.

Stangerlin et al. [11] conducting research on wood of Eucalyptus dunnii in order to investigate the effect of positioning the fiber (radial and tangential) for obtaining the toughness and impact strength. The authors concluded that the positioning in the radial direction provided higher values of impact strength and toughness when compared with the 
specimens positioned in the tangential direction.

Stangerlin et al. [12] studied the impact strength of Eucalyptus saligna and Eucalyptus botrioides wood species, concluding, among other, not possible to estimate the toughness of the wood through the bulk density.

Beltrame et al. [13] evaluated, using the pendulum Charpy impact strength, coefficient of resilience and dynamic dimension wood-horse whips (Luehea divaricata Mart. Mart et Zucc.) Tested under equilibrium conditions at $12 \%$ moisture content $\left(0.628 \mathrm{~g} / \mathrm{cm}^{3}\right)$ and saturated in water $(1.076$ $\mathrm{g} / \mathrm{cm}^{3}$ ), using trees from two physiographic regions of the state of Rio Grande do Sul, were investigated also in this study the influence of the position (bone and shell) and direction (tangential and radial) of the specimen in the tests for obtaining those mechanical properties. The results showed that the wood-horse whips evaluated in saturated condition showed higher impact strength and toughness (37.03J), absorbing much of the applied load on it when compared to the strength and toughness at $12 \%$ of moisture content $(28.87 \mathrm{~J})$, and with respect to the position and direction of the orientation of the specimens in obtaining the toughness, the results of statistical analysis showed equivalence, implying not significant effect of these two factors on the toughness of wood-horse whips.

Beltrame et al. [14] investigated the influence of moisture content $(12 \%$ and saturated) and the impact strength of Walnut pecan (Carya illinoinensis), from two physiographic regions of the state of Rio Grande do Sul (Brazil). Specimens were also prepared and tested for impact strength directions, radial and tangential positions and withdrawal, near the spinal cord and the next shell. As for the energy absorbed or toughness, saturated parts showed higher $(7.774 \mathrm{~kg} \mathrm{~m})$ compared the toughness of woods with $12 \%$ of moisture content $(2.976 \mathrm{~kg} \mathrm{~m})$, about $161 \%$ higher on average.

In order to generate subsidies to expand the knowledge about the behavior of wood when requested by impact loads, this study aimed to investigate, using polynomial regression models, the possibility of estimating the toughness as a function of density and bending strength, making use of fifteen species of wood chosen and equally distributed in five classes of strength (C-20, C-30 to C-60) presented by the Brazilian standard ABNT NBR 7190 [15].

\section{Material and Methods}

Table 1 shows the species of wood used in this research, being duly grouped in strength classes of Brazilian standard ABNT NBR 7190 [15].

The toughness tests $(W)$ were performed according to the procedures and assumptions for the calculation of ASTM D5536-94 [16], using the Charpy pendulum, and the apparent density $\left(\rho_{12 \%}\right)$ and the bending strength $\left(f_{m}\right)$ followed the recommendations of the Brazilian standard ABNT NBR 7190 [15]. We used 12 specimens per species of wood required for each type of test.
Table 1. Wood species investigated

\begin{tabular}{ccc}
\hline $\begin{array}{c}\text { Strength } \\
\text { Classes }\end{array}$ & Vulgar Name & Scientific Name \\
\hline & Cedro Doce & Cedrela sp \\
C-20 & Cedrona & Cedrelinga catenaeformis \\
& Quarubarana & Erisma uncinatum \\
& Canafístula & Peltophorum dubium \\
C-30 & Cedro Amargo & Cedrela odorata \\
& Cupiúba & Goupia glabra \\
& Branquilho & Terminalia sp \\
C-40 & Cafearana & Andira sp \\
& Louro Preto & Ocotea sp \\
& Castelo & Gossypiospermum \\
& Angico Preto & Piptadenia macrocarpa \\
C-50 & Catanudo & Calophyllum sp \\
& Garapa & Apuleia leiocarpa \\
& Champanhe & Dipteryx odorata \\
& Sucupira & Diplotropis sp \\
\hline
\end{tabular}

In order to relate the toughness with apparent density ( $W$; $\left.\rho_{12 \%}\right)$ and toughness with strength in bending $\left(W ; \mathrm{f}_{\mathrm{m}}\right)$, were tested polynomial regression models of linear, quadratic and cubic approximations. Therefore, the regression models evaluated through analysis of variance (ANOVA) were considered at a significance level of $5 \%$, with the null hypothesis of no significance setting provided by the model $\left(\beta_{i}=0\right)$ and the significance $\left(\beta_{i} \neq 0\right)$ adjustment obtained as alternative hypothesis. P-value less than the level of significance set implies rejecting $\mathrm{H}_{0}$ (Fdados $>$ Fcrítico), accepting it otherwise.

\section{Results}

Table 2. Results of the properties from C-20 strength class

\begin{tabular}{|c|c|c|c|}
\hline & \multicolumn{3}{|c|}{ Cedro Doce } \\
\hline & $\begin{array}{c}\rho_{12 \%} \\
\left(\mathrm{~g} / \mathbf{c m}^{3}\right) \\
\end{array}$ & $\begin{array}{c}f_{m} \\
\left(10^{5} \mathrm{~N} / \mathbf{m}^{2}\right) \\
\end{array}$ & $W(J)$ \\
\hline $\bar{x}$ & 0.50 & 567 & 51 \\
\hline$C v$ & 12 & 19 & 23 \\
\hline Min. & 0.38 & 470 & 32 \\
\hline \multirow[t]{3}{*}{ Máx. } & 0.65 & 820 & 75 \\
\hline & \multicolumn{3}{|c|}{ Cedrona } \\
\hline & $\begin{array}{c}\rho_{12 \%} \% \\
\left(\mathrm{~g} / \mathbf{c m}^{3}\right)\end{array}$ & $\begin{array}{c}\mathbf{f}_{\mathrm{m}} \\
\left(10^{5} \mathrm{~N} / \mathrm{m}^{2}\right)\end{array}$ & $W(J)$ \\
\hline $\bar{x}$ & 0.57 & 572 & 46 \\
\hline$C v$ & 7 & 15 & 17 \\
\hline Min. & 0.50 & 410 & 34 \\
\hline \multirow[t]{3}{*}{ Máx. } & 0.64 & 650 & 59 \\
\hline & \multicolumn{3}{|c|}{ Quarubarana } \\
\hline & $\begin{array}{c}\rho_{12 \%} \\
\left(\mathrm{~g} / \mathbf{c m}^{3}\right)\end{array}$ & $\begin{array}{c}\mathbf{f}_{\mathrm{m}} \\
\left(10^{5} \mathrm{~N} / \mathrm{m}^{2}\right)\end{array}$ & $W(\mathbf{J})$ \\
\hline $\bar{x}$ & 0.54 & 650 & 43 \\
\hline$C v$ & 10 & 13 & 20 \\
\hline Min. & 0.47 & 530 & 29 \\
\hline Máx. & 0.67 & 790 & 56 \\
\hline
\end{tabular}


Table 2 to 6 presents the results of physical and mechanical properties of wood evaluated, where $\bar{x}$ is the sample mean, $C v$ is the coefficient of variation and Min and Max the least and greatest values found.

Table 3. Results of the properties from C-30 strength class

\begin{tabular}{|c|c|c|c|}
\hline & \multicolumn{3}{|c|}{ Canafístula } \\
\hline & $\begin{array}{c}\rho_{12 \%} \\
\left(\mathrm{~g} / \mathrm{cm}^{3}\right)\end{array}$ & $\begin{array}{c}\mathbf{f}_{\mathrm{m}} \\
\left(10^{5} \mathrm{~N} / \mathrm{m}^{2}\right)\end{array}$ & $W(\mathrm{~J})$ \\
\hline $\bar{x}$ & 0.86 & 887 & 108 \\
\hline$C v$ & 9 & 12 & 22 \\
\hline Min. & 0.74 & 720 & 86 \\
\hline \multirow[t]{3}{*}{ Máx. } & 0.95 & 1090 & 129 \\
\hline & \multicolumn{3}{|c|}{ Cedro Amargo } \\
\hline & $\begin{array}{c}\rho_{12 \%} \\
\left(\mathbf{g} / \mathbf{c m}^{3}\right) \\
\end{array}$ & $\begin{array}{c}\mathbf{f}_{\mathrm{m}} \\
\left(10^{5} \mathrm{~N} / \mathrm{m}^{2}\right)\end{array}$ & $W(\mathrm{~J})$ \\
\hline $\bar{x}$ & 0.50 & 667 & 52.25 \\
\hline$C v$ & 4 & 14 & 25 \\
\hline Min. & 0.46 & 550 & 31 \\
\hline \multirow[t]{3}{*}{ Máx. } & 0.53 & 870 & 73 \\
\hline & \multicolumn{3}{|c|}{ Cupiúba } \\
\hline & $\begin{array}{c}\rho_{12 \%} \\
\left(\mathrm{~g} / \mathbf{c m}^{3}\right)\end{array}$ & $\begin{array}{c}\mathbf{f}_{\mathrm{m}} \\
\left(10^{5} \mathrm{~N} / \mathrm{m}^{2}\right)\end{array}$ & $W(\mathrm{~J})$ \\
\hline $\bar{x}$ & 0.85 & 900 & 75 \\
\hline$C v$ & 4 & 16 & 17 \\
\hline Min. & 0.77 & 710 & 57 \\
\hline Máx. & 0.90 & 1140 & 96 \\
\hline
\end{tabular}

Table 4. Results of the properties from C-40 strength class

\begin{tabular}{|c|c|c|c|}
\hline & \multicolumn{3}{|c|}{ Branquilho } \\
\hline & $\begin{array}{c}\rho_{12 \%} \\
\left(\mathrm{~g} / \mathbf{c m}^{3}\right) \\
\end{array}$ & $\begin{array}{c}\mathbf{f}_{\mathrm{m}} \\
\left(10^{5} \mathrm{~N} / \mathrm{m}^{2}\right)\end{array}$ & $W(\mathbf{J})$ \\
\hline $\bar{x}$ & 0.81 & 843 & 103 \\
\hline$C v$ & 6 & 13 & 18 \\
\hline Min. & 0.73 & 660 & 77 \\
\hline \multirow[t]{3}{*}{ Máx. } & 0.89 & 1030 & 142 \\
\hline & \multicolumn{3}{|c|}{ Cafearana } \\
\hline & $\begin{array}{c}\rho_{12 \%} \\
\left(\mathbf{g} / \mathbf{c m}^{3}\right)\end{array}$ & $\begin{array}{c}\mathbf{f}_{\mathrm{m}} \\
\left(10^{5} \mathrm{~N} / \mathrm{m}^{2}\right)\end{array}$ & $W(\mathrm{~J})$ \\
\hline $\bar{x}$ & 0.67 & 910 & 86 \\
\hline$C v$ & 8 & 19 & 18 \\
\hline Min. & 0.61 & 720 & 65 \\
\hline \multirow[t]{3}{*}{ Máx. } & 0.78 & 1270 & 119 \\
\hline & \multicolumn{3}{|c|}{ Louro Preto } \\
\hline & $\begin{array}{c}\rho_{12 \%} \\
\left(\mathrm{~g} / \mathrm{cm}^{3}\right)\end{array}$ & $\begin{array}{c}\mathbf{f}_{\mathrm{m}} \\
\left(10^{5} \mathrm{~N} / \mathrm{m}^{2}\right)\end{array}$ & $W(\mathrm{~J})$ \\
\hline $\bar{x}$ & 0.68 & 1046 & 74 \\
\hline$C v$ & 4 & 21 & 21 \\
\hline Min. & 0.64 & 820 & 52 \\
\hline Máx. & 0.74 & 1370 & 99 \\
\hline
\end{tabular}

Table 5. Results of the properties from C-50 strength class

\begin{tabular}{|c|c|c|c|}
\hline & \multicolumn{3}{|c|}{ Castelo } \\
\hline & $\begin{array}{c}\rho_{12} \% \\
\left(\mathrm{~g} / \mathrm{cm}^{3}\right)\end{array}$ & $\begin{array}{c}f_{m} \\
\left(10^{5} \mathrm{~N} / \mathrm{m}^{2}\right)\end{array}$ & $W(\mathrm{~J})$ \\
\hline $\bar{x}$ & 0.76 & 1030 & 144 \\
\hline$C v$ & 9 & 6 & 19 \\
\hline Min. & 0.60 & 892 & 115 \\
\hline \multirow[t]{3}{*}{ Máx. } & 0.88 & 1119 & 199 \\
\hline & \multicolumn{3}{|c|}{ Angico Preto } \\
\hline & $\begin{array}{c}\rho_{12} \% \\
\left(\mathrm{~g} / \mathbf{c m}^{3}\right)\end{array}$ & $\begin{array}{c}\mathbf{f}_{\mathrm{m}} \\
\left(10^{5} \mathrm{~N} / \mathrm{m}^{2}\right)\end{array}$ & $W(J)$ \\
\hline $\bar{x}$ & 0.89 & 1223 & 140 \\
\hline$C v$ & 7 & 24 & 20 \\
\hline Min. & 0.80 & 900 & 85 \\
\hline \multirow[t]{3}{*}{ Máx. } & 1.03 & 1770 & 159 \\
\hline & \multicolumn{3}{|c|}{ Catanudo } \\
\hline & $\begin{array}{c}\rho_{12} \% \\
\left(\mathbf{g} / \mathbf{c m}^{3}\right) \\
\end{array}$ & $\begin{array}{c}f_{m} \\
\left(10^{5} \mathrm{~N} / \mathrm{m}^{2}\right)\end{array}$ & $W(\mathrm{~J})$ \\
\hline $\bar{x}$ & 0.80 & 885 & 126 \\
\hline$C v$ & 5 & 12 & 19 \\
\hline Min. & 0.74 & 690 & 83 \\
\hline Máx. & 0.87 & 1040 & 165 \\
\hline
\end{tabular}

Table 6. Results of the properties from C-60 strength class

\begin{tabular}{|c|c|c|c|}
\hline & \multicolumn{3}{|c|}{ Garapa } \\
\hline & $\begin{array}{c}\rho_{12 \%} \\
\left(\mathrm{~g} / \mathrm{cm}^{3}\right)\end{array}$ & $\begin{array}{c}\mathbf{f}_{\mathrm{m}} \\
\left(10^{5} \mathrm{~N} / \mathrm{m}^{2}\right)\end{array}$ & $W(\mathbf{J})$ \\
\hline $\bar{x}$ & 0.92 & 1255 & 127 \\
\hline$C v$ & 6 & 15 & 19 \\
\hline Min. & 0.85 & 960 & 93 \\
\hline \multirow[t]{3}{*}{ Máx. } & 1.03 & 1550 & 172 \\
\hline & \multicolumn{3}{|c|}{ Champanhe } \\
\hline & $\begin{array}{c}\rho_{12 \%} \\
\left(\mathrm{~g} / \mathrm{cm}^{3}\right) \\
\end{array}$ & $\begin{array}{c}\mathbf{f}_{\mathrm{m}} \\
\left(10^{5} \mathrm{~N} / \mathrm{m}^{2}\right)\end{array}$ & $W(\mathbf{J})$ \\
\hline $\bar{x}$ & 1.09 & 1581 & 203 \\
\hline$C v$ & 3 & 9 & 12 \\
\hline Min. & 1.04 & 1320 & 167 \\
\hline \multirow[t]{3}{*}{ Máx. } & 1.14 & 1820 & 243 \\
\hline & \multicolumn{3}{|c|}{ Sucupira } \\
\hline & $\begin{array}{c}\rho_{12 \%} \\
\left(\mathrm{~g} / \mathbf{c m}^{3}\right)\end{array}$ & $\begin{array}{c}\mathbf{f}_{\mathrm{m}} \\
\left(10^{5} \mathrm{~N} / \mathrm{m}^{2}\right)\end{array}$ & $W(\mathbf{J})$ \\
\hline $\bar{x}$ & 1.10 & 1465 & 168 \\
\hline$C v$ & 6 & 15 & 14 \\
\hline Min. & 0.99 & 930 & 136 \\
\hline Máx. & 1.22 & 1660 & 214 \\
\hline
\end{tabular}

Figures 1 and 2 illustrate the results of adjustments by the regression models for toughness as a function of density and bending strength, respectively. 


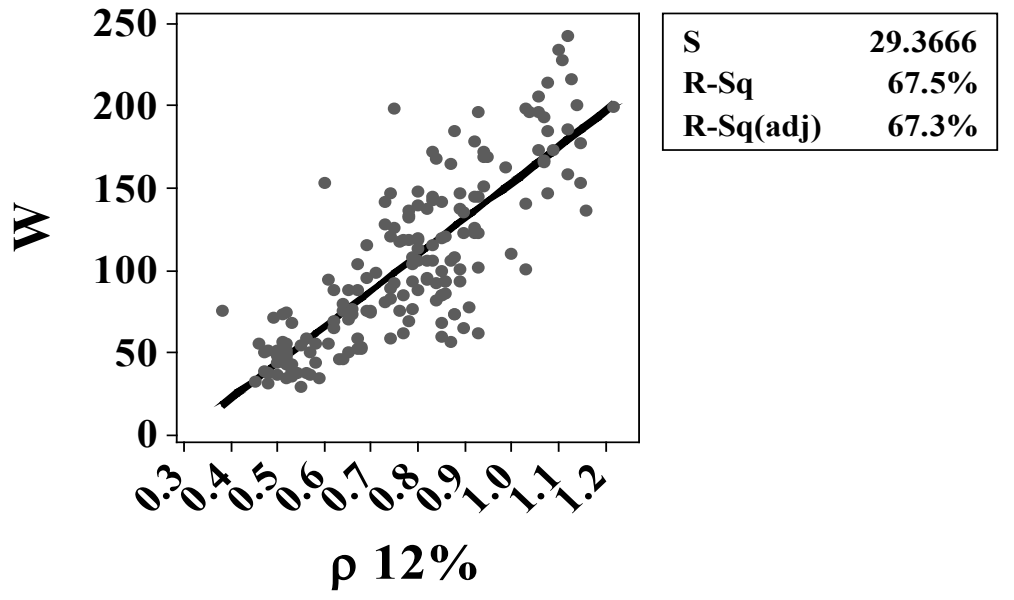

(a)

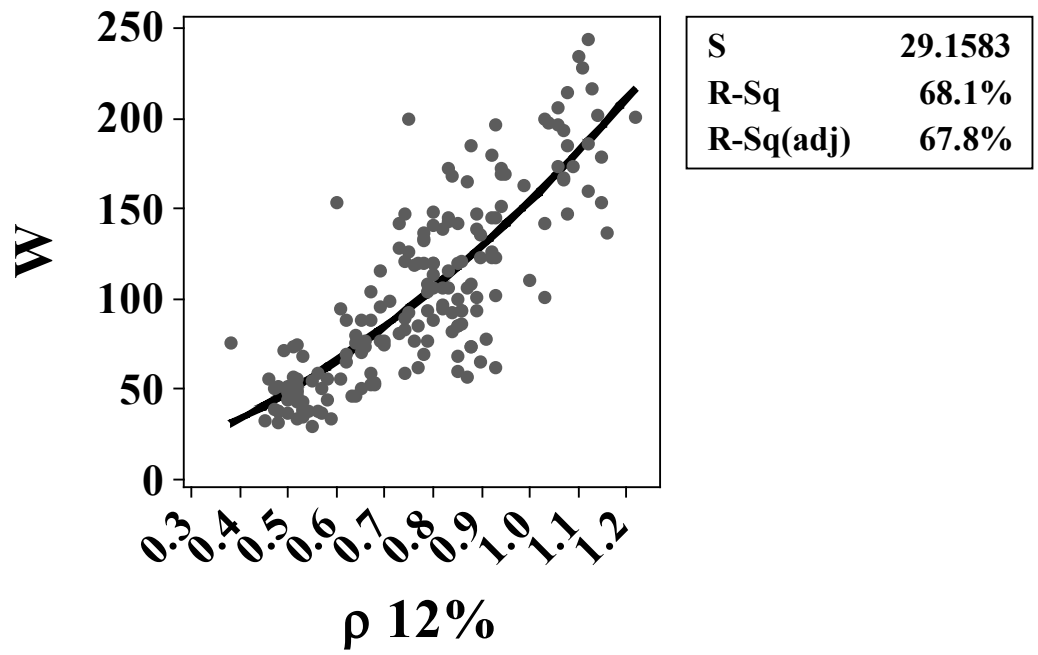

(b)

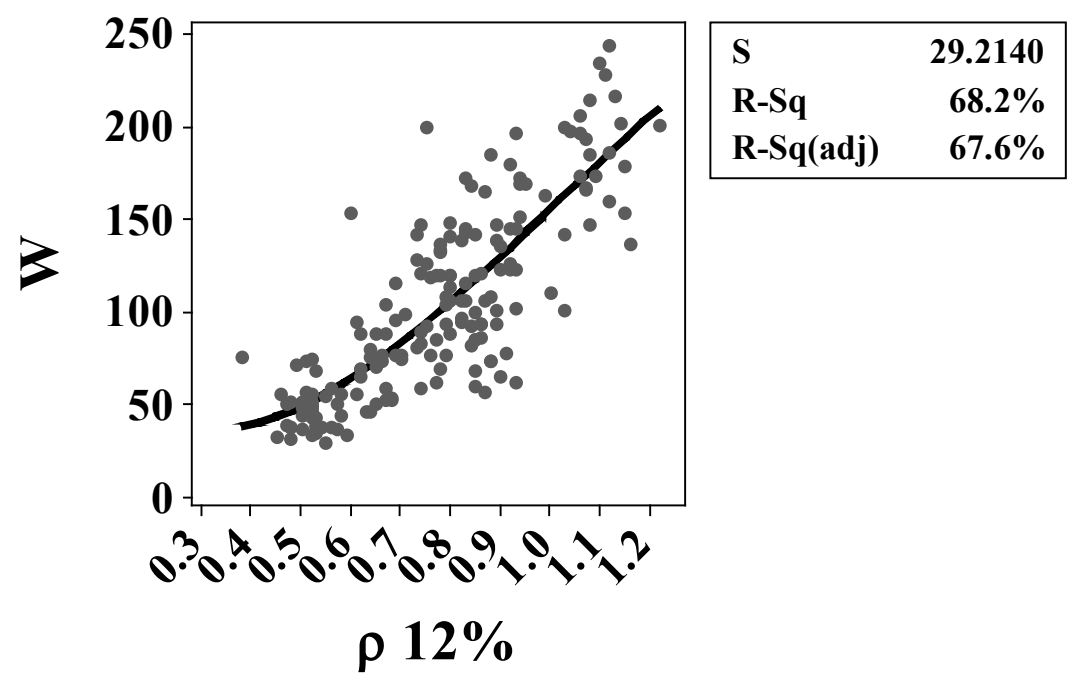

(c)

Figure 1. Adjustments by polynomial regression models: (a) linear, (b) a quadratic and cubic (c) for toughness as a function of apparent density 


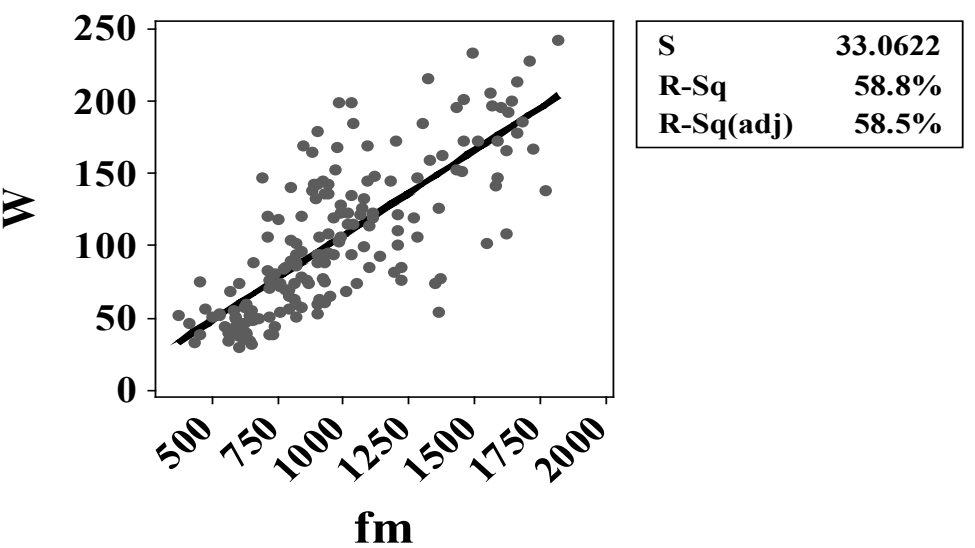

(a)

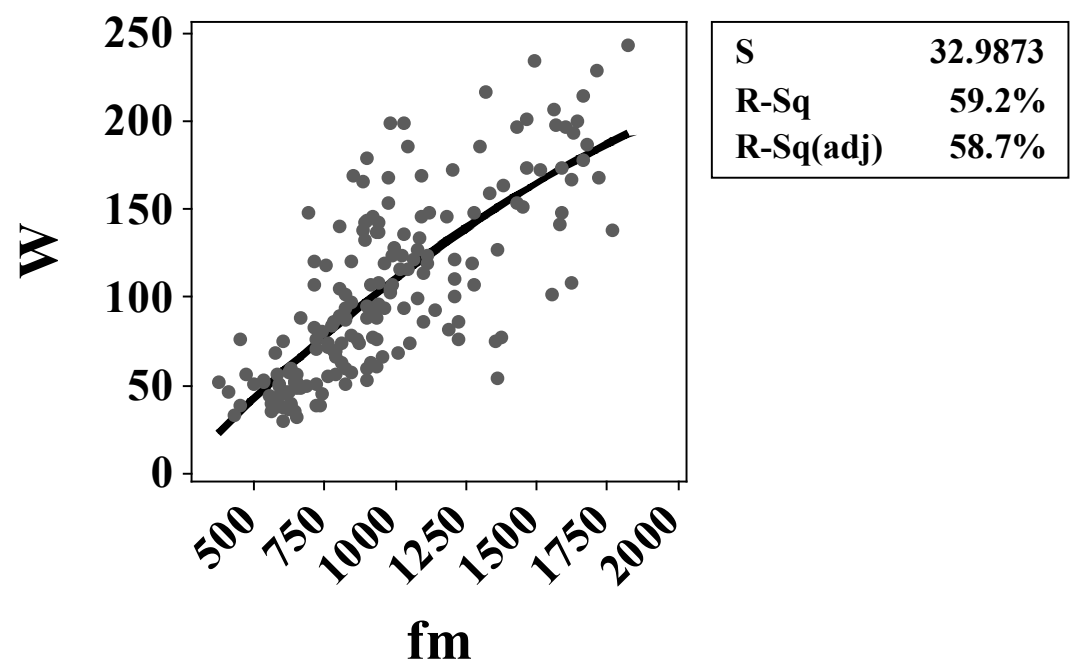

(b)

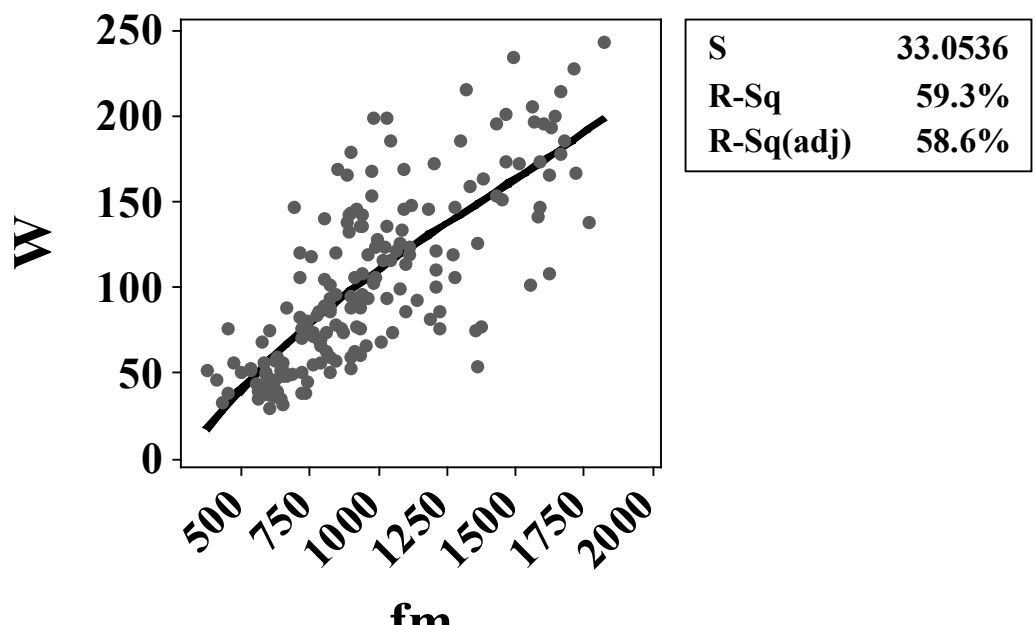

(c)

Figure 2. Adjustments by polynomial regression models: (a) linear, (b) a quadratic and cubic (c) for toughness as a function of the strength in bending 
Table 7. Adjustments obtained from regression models

\begin{tabular}{ccc}
\hline & $\boldsymbol{W} \times \boldsymbol{\rho}$ & \\
\hline Adjust & Equation & $\mathbf{R}^{\mathbf{2} \text { (adj.) }}$ \\
\hline $1^{\circ}$ & $W=-64,80+217,9 \cdot \rho_{12 \%}$ & $63,30 \%$ \\
$2^{\circ}$ & $W=-5,76+57,91 \cdot \rho_{12 \%}+101,8 \cdot \rho_{12 \%}{ }^{2}$ & $67,80 \%$ \\
$3^{\circ}$ & $W=70,90-255,8 \cdot \rho_{12 \%}+509,40 \cdot \rho_{12 \%}{ }^{2}-169,1 \cdot \rho_{12 \%}{ }^{3}$ & $67,60 \%$ \\
\hline & $W \times f_{\mathrm{m}}$ & \\
\hline Adjust & Equação & $\mathbf{R}^{2}$ (adj.) \\
\hline $1^{\circ}$ & $W=-11,04+0,1181 \cdot f_{m}$ & $58,50 \%$ \\
$2^{\circ}$ & $W=-68,80+0,2743 \cdot f_{m}-0,000126 \cdot f_{m}{ }^{2}+0,000003 \cdot f_{m}{ }^{3}$ & $58,60 \%$ \\
\hline $3^{\circ}$ & $W$ & $58,70 \%$ \\
\hline
\end{tabular}

Table 8. ANOVA results of the regression models for toughness as a function of apparent density

\begin{tabular}{cccccc}
\hline \multicolumn{6}{c}{ Linear Model } \\
\hline Source & DF & SS & MS & $\mathbf{F}_{\text {dados }}$ & P-valor \\
\hline Regression & 1 & 318545 & 318545 & 369,37 & 0,000 \\
Error & 178 & 153507 & 862 & & \\
Total & 179 & 472052 & & & \\
\hline \multicolumn{5}{c}{ Quadratic Model } \\
\hline Source & DF & SS & MS & $\mathbf{F}_{\text {dados }}$ & P-valor \\
\hline Regression & 2 & 321565 & 160783 & 189,11 & 0,000 \\
Error & 177 & 150487 & 850 & & \\
Total & 179 & 472052 & & & \\
\hline \multicolumn{7}{c}{ Cúbic Model } \\
\hline Source & DF & SS & MS & $\mathbf{F}_{\text {dados }}$ & P-valor \\
\hline Regression & 3 & 321843 & 107281 & 125,70 & 0,000 \\
Error & 176 & 150209 & 853 & & \\
Total & 179 & 472052 & & & \\
\hline
\end{tabular}

Table 9. ANOVA results of the regression models for toughness as a function of bending strength

\begin{tabular}{cccccc}
\hline \multicolumn{6}{c}{ Linear Model } \\
\hline Source & DF & SS & MS & $\mathbf{F}_{\text {dados }}$ & P-valor \\
\hline Regression & 1 & 277479 & 277479 & 253,84 & 0,000 \\
Error & 178 & 194573 & 1093 & & \\
Total & 179 & 472052 & & & \\
\hline \multicolumn{5}{c}{ Quadratic Model } \\
\hline Source & DF & SS & MS & $\mathbf{F}_{\text {dados }}$ & P-valor \\
\hline Regression & 2 & 279448 & 139724 & 128,40 & 0,000 \\
Error & 177 & 192602 & 1088 & & \\
Total & 179 & 472052 & & & \\
\hline \multicolumn{5}{c}{ Cúbic Model } & \\
\hline Source & DF & SS & MS & $\mathbf{F}_{\text {dados }}$ & P-valor \\
\hline Regression & 3 & 279765 & 93255,1 & 85,36 & 0,000 \\
Error & 176 & 192287 & 1092,5 & & \\
Total & 179 & 472052 & & & \\
\hline
\end{tabular}

Table 7 shows the equations and the corresponding adjusted coefficient of determination $\left(\mathrm{R}^{2}\right.$ (adj.)), and Tables 8 and 9 the results of analysis of variance for toughness as a function of apparent density and strength in bending, respectively; DF is the degrees of freedom, SS is the sum of squares and MS is the average of the square.

P-values obtained by ANOVA (Tables 8 and 9) are both higher than the level of significance set $\left(\mathrm{F}_{\text {dados }}>\mathrm{F}_{\text {crítico }}\right)$, all regression models should be accepted. Adjustments tested, the quadratic polynomial model proved to be the better for having the largest value of $\mathrm{R}^{2}$ (adj.) for both adjustments (toughness as function of density and bending strength).

The $\mathrm{R}^{2}$ (adj.) coefficients obtained by toughness as a function of apparent density was shown to be more accurate, indicating that the best estimate for the toughness of woods investigated.

\section{Conclusions}

The results of the regression models with the use of fifteen species of wood, conveniently chosen to cover the five classes of strength contemplated by the Brazilian standard ABNT NBR 7190:1997, indicate possible to estimate the toughness, revealing the quadratic polynomial model as function of the apparent density as the most suitable. Thus, the toughness of wood can be estimated with knowledge of the results of apparent density with the quadratic equation obtained by the least squares fit.

\section{REFERENCES}

[1] Kollmann, F. F. P.; Cotê, W. A. Principles of wood science and technology: Solid wood. Springer, Verlag, 263p., 1968.

[2] Askeland, D. R. The science and engineering of materials. 3ed., Bostons, PWS Publishing Company, 1994.

[3] Van Vlack, L. H. Elements of materials science and engineering. 6ed., Addison, Wesley Publishing Company, 1989.

[4] Schneider, M. H.; Phillips, J. G.; Brebner, K. I.; Tingley, D. A. 
Toughness of polymer impregnated sugar maple at two moisture contents. Forest products Journal, v. 39, n. 6, p. 11-14, 1989.

[5] Fiorelli, J.; Dias, A. A. Glulam beams reinforced with FRP externally-bonded: theoretical and experimental evaluation. Materials and Structures, v. 44, p. 1431-1440, 2011.

[6] Carreira, M. R.; Segundinho, P. G. A.; Rocco Lahr, F. A.; Dias, A. A.; Calil Jr., C. Bending stiffness evaluation of Teca and Guajará lumber through tests of transverse and longitudinal vibration. Acta Scientiarum Technology (Impresso), v. 34, p. 27-32, 2012.

[7] Christoforo, A. L.; Ribeiro Filho, S. L. M.; Panzera, T. H.; Rocco, F. A. L. Metodologia para o cálculo dos módulos de elasticidade longitudinal e transversal em vigas de madeira de dimensões estruturais. Ciência Rural (UFSM. Impresso), v. 43, p. $610,2013$.

[8] Stolf, D. O. Tenacidade da madeira. 2000, 1101 p. Dissertação (Mestrado em Interunidades em Ciência dos Materiais). Escola de Engenharia de São Carlos da Universidade de São Paulo. São Carlos - SP, 2000.

[9] Souza, J. T.; Machado, W. G.; Beltrame, R.; Modes, K. S.; Vivian, M. A.; Haselein, C. R. Estudo do comportamento da flexão dinâmica em função da massa específica da madeira de Araucaria angustifolia (Bertol.) Kuntze. XVIII Congresso de Iniciação Científica (CIC), XI Encontro de Pós-Graduação (ENPOS) e a I Mostra Científica. Anais... CD ROM. Universidade Federal de Pelotas, Pelotas - RS, 5 p., 2009.

[10] Pazos, G. B.; Morales, R. P. Z.; Candelaria, V. R. O. Correlación de los resultados de impacto o tenacidad de 16 maderas mexicanas utilizando dos métodos de prueba. Revista Madera y Bosques, v. 9, n. 1, p. 55-70, 2003.

[11] Stangerlin, D. M.; Melo, R. R.; Domingues, J. M. X. Determinação da resistência ao impacto para as madeiras de Eucalyptus dunnii, Corymbia citriodora e Pouteria pachycarpa. In: XI Encontro Brasileiro em Madeira e Estruturas de Madeira. Londrina. Anais... CD ROM, Londrina, 2008.

[12] Stangerlin, D. M.; Gatto, D. A.; Domingues, J. M. X.; Calegari, L.; Muller, M. T. Avaliação da resistência ao impacto para as madeiras de Eucalyptus botrioides e Eucalyptus saligna. In: X Congresso Florestal Estadual, Nova Prata, RS. Anais... CD ROM, 2008.

[13] Beltrame, R.; Gatto, D. A.; Modes, K. S.; Stangerlin, D. M.; Trevisan, R.; Haselein, C. R. Resistência ao impacto da madeira de açoita-cavalo em diferentes condições de umidade. Cerne (UFLA), v. 16, p. 499-504, 2010.

[14] Beltrame, R.; Mattos, B. D.; Gatto, D. A.; Lazarotto, M.; Haselein, C. R. ; Santini, E. J. Resistência ao impacto da madeira de nogueira-pecã em diferentes condições de umidade. Ciência Rural, v.42, n.9, 2012.

[15] Associação Brasileira de Normas Técnicas ABNT NBR 7190. Projeto de estruturas de madeira. Rio de Janeiro, 1997.

[16] American Society for Testing and Materials ASTM D5536-94. Standard methods of testing small clear specimens of timber. Philadelphia, 1995. 